\title{
Implante de biomateriais e a consolidação óssea em cadelas submetidas à ovariossalpingo-histerectomia
}

\author{
[Influence of biomaterials on the bony consolidation in spayed female dogs] \\ A.I. Roque-Rodriguez ${ }^{1}$, S.M. Baraldi-Artoni ${ }^{1}{ }^{*}$, D. Oliveira ${ }^{2}$ \\ ${ }^{1}$ Faculdade de Ciências Agrárias e Veterinárias - UNESP \\ Via de Acesso Prof. Paulo Donato Castellane, s/n, \\ 14884-900 - Jaboticabal, SP \\ ${ }^{2}$ Unidade Acadêmica de Garanhuns - UFRPE - Garanhuns, PE
}

\begin{abstract}
RESUMO
Avaliou-se a hidroxiapatita com alandronato e hidroxiapatita com colágeno na aceleração da consolidação óssea do rádio de cadelas adultas submetidas à ovariossalpingo-histerectomia (OSH). Utilizaram-se 14 cadelas adultas, distribuídas aleatoriamente em dois grupos: grupo-controle e grupo OSH (submetidas à $\mathrm{OSH}$ ). Quatro meses após a OSH, as cadelas dos dois grupos foram submetidas à cirurgia para produção de uma falha óssea de $4 \mathrm{~mm}$ de diâmetro nos terços distal e proximal do rádio. No terço distal do membro direito, foi utilizada a hidroxiapatita com alandronato e, no membro esquerdo, a hidroxiapatita com colágeno; no terço proximal, não se utilizou nenhum biomaterial. Houve retardo na consolidação das falhas ósseas nas cadelas submetidas à OSH comparadas com as não submetidas. A hidroxiapatita com alandronato acelerou o processo de reparação e, em todos os animais dos dois grupos, a densidade óssea foi significativamente maior no terço distal onde foi implantada. Os dois biomateriais apresentaram biocompatibilidade, constatada pela ausência de reação inflamatória ou outra reação indesejável.
\end{abstract}

Palavras-chave: cadela, ovariossalpingo-histerectomia, hidroxiapatita, alandronato, colágeno, calo ósseo

\begin{abstract}
The hydroxyapatite with alendronate and hydroxyapatite with collagen were evaluated in the acceleration of the bony consolidation of adult spayed bitch radius. For that, 14 adult bitches were distributed in two groups (control and spayed). Four months after ovariohysterectomy, the groups were submitted to the surgery for production of a $4 \mathrm{~mm}$ diameter bony flaw in the distal and proximal third regions of the radius. In the distal region of the right thoracic limb, hydroxyapatite with alendronate was used. In the distal region of the left thoracic limb, hydroxyapatite with collagen was used. Any biomaterial was used in proximal part of the limb. There was a retard in bony flaws consolidation in the spayed bitches. Hydroxyapatite with alendronate showed better result, since the place it was implanted considerably increased the bony formation. Both biomaterials presented biocompatibility, verified by the absence of inflammatory reaction or other undesirable reaction.
\end{abstract}

Keywords: bitch, ovariohysterectomy, hydroxyapatite, alendronate, collagen, fracture callus

\section{INTRODUÇ̃̃̃O}

As fraturas de ossos longos dos membros dos animais domésticos de pequeno porte constituem um importante campo da patologia cirúrgica e representam grande parte dos casos atendidos em ambulatórios. $\mathrm{O}$ traumatismo apresenta considerável variação de formas, além de dificuldades no seu tratamento, face às particularidades dos casos, ao tempo de

Recebido em 26 de fevereiro de 2009

Aceito em 15 de outubro de 2009

*Autor para correspondência (corresponding author)

E-mail: smbart@fcav.unesp.br 
resolução, e à necessidade de atuação de profissionais com conhecimento especializado. Após a redução das fraturas, é importante que a cicatrização óssea ocorra o mais rápido possível, a fim de evitar a atrofia muscular por desuso (Wheeler et al., 2000).

Os estrógenos são estimuladores da formação do tecido ósseo, pois influenciam o aparecimento e o desenvolvimento dos centros de ossificação. Nos casos de desenvolvimento deficiente das gônadas ou de ovariossalpingo-histerectomia (OSH) de animais em crescimento, as cartilagens epifisárias permanecem por mais tempo, de modo que o indivíduo atinge estatura acima da normal (Junqueira e Carneiro, 1999). Depois da interrupção da função ovárica, observa-se diminuição da massa óssea (que caracteriza a osteoporose) com desorganização da microarquitetura trabecular e, consequentemente, aumento do risco da ocorrência de fraturas ou refraturas (Souza et al., 2003).

Malluche et al. (1988) estudaram, por meio de análises histomorfométricas, a remodelação óssea em cães da raça Beagle, com perda significativa do osso quatro meses após a OSH, e observaram que a insuficiência osteoblástica é a responsável pelo balanço negativo da manutenção do osso e pela osteopenia.

Alguns compostos de hidroxiapatita com colágeno foram desenvolvidos como biomateriais em potencial para a reconstrução óssea por apresentarem uma composição similar à do osso (Du et al., 1998; Bakos et al., 1999). O aspecto mais importante na utilização de colágeno na forma de compostos com biocerâmicas é o fato de esta proteína ser a matriz em que os sais de fosfato de cálcio se depositam para dar origem ao tecido ósseo (Mann et al., 1989). Os efeitos biológicos do alandronato derivam da forte afinidade pelo osso, o que possibilita uma interação direta com osteoblastos, aumentando a formação e, com os osteoclastos, diminuindo a reabsorção óssea, por meio de uma variedade de ciclos bioquímicos (Masarachia, 1996).

A densitometria óptica é um método que não depende de equipamentos sofisticados, é viável e de fácil execução, custo baixo, altamente confiável, não invasivo e que permite a realização de análises qualitativas e quantitativas do conteúdo e da densidade mineral. Com esta técnica, eliminam-se possíveis interpretações subjetivas que ocorrem durante a avaliação radiográfica, pois esta apresenta baixa sensibilidade para o diagnóstico precoce de perdas ósseas, que são detectadas somente quando excedem $30 \%$ a $50 \%$ e, por muitas vezes, só são perceptíveis no momento em que ocorre uma fratura ou refratura (Gluer e Genant, 2002).

Em medicina humana, estudos realizados em algumas espécies animais têm o intuito de analisar as alterações ósseas após a OSH e correlacioná-las com as da mulher. $\mathrm{O}$ efeito da deficiência hormonal sobre a consolidação das fraturas em cadelas pode ser diferente da mulher e de outros animais e, portanto, análises comparativas entre espécies frente às influências hormonais sobre a consolidação óssea requerem estudos criteriosos.

O objetivo deste trabalho foi avaliar os efeitos da hidroxiapatita com alandronato e da hidroxiapatita com colágeno na consolidação óssea do rádio de cadelas adultas submetidas à ovariossalpingo-histerectomia.

\section{MATERIAL E MÉTODOS}

Utilizaram-se 14 cadelas adultas (dois a seis anos de idade), de porte médio $(14 \pm 1,3 \mathrm{~kg})$, hígidas por ocasião de sua escolha, com base nos resultados de exame clínico detalhado e testes hematológicos, coproparasitológicos e de urina. As cadelas foram vacinadas e vermifugadas, alimentadas com ração balanceada e água à vontade. Depois de aclimatadas por um período de um mês, antes do início do experimento, foram distribuídas aleatoriamente em dois grupos: grupo-controle e grupo das submetidas à ovariossalpingo-histerectomia (OSH).

Quatro meses após a realização da ovariossalpingo-histerectomia, as cadelas dos dois grupos foram submetidas à cirurgia para produção de duas falhas ósseas de $4 \mathrm{~mm}$ de diâmetro no terço proximal e distal do rádio de ambos os membros. No terço distal do membro direito, foi implantada a hidroxiapatita com alandronato, e no membro esquerdo, a hidroxiapatita com colágeno. No terço proximal, a falha óssea não foi preenchida por biomateriais, estabelecendo-se como controle de ambos os grupos. 
Foram utilizados dois tipos de biomateriais, na mesma quantidade $(0,12 \mathrm{~g})$, esterilizados em óxido de etileno: 1) hidroxiapatita sintética $(\text { HAP-91 })^{1}$ com alandronato $(4,2 \mathrm{mg} \quad \mathrm{de}$ alandronato e $200 \mathrm{mg}$ de hidroxiapatita); 2) hidroxiapatita sintética com colágeno (COL.HAP-91) ${ }^{1}$, nas concentrações de $75 \%$ de hidroxiapatita e $25 \%$ de colágeno.

Nas cadelas dos dois grupos, foram feitas avaliações clínicas diárias, até o $20^{\circ}$ dia do pósoperatório; os exames laboratoriais hemograma, dosagens de fosfatase alcalina, cálcio, fósforo e magnésio - foram realizados no quarto mês pós-OSH.

Empregaram-se o anti-inflamatório não esteroidal à base de flunixin meglumine ${ }^{2}$ e antibioticoterapia à base de ampicilina sódica e benzatínica. Foram realizados curativos e utilizaram-se colar elisabetano. Os exercícios físicos foram restringidos, mas o uso dos membros foi permitido.

As avaliações radiográficas foram realizadas nos dias $0,30,60,90$ e 120 do período pósoperatório. $\mathrm{O}$ aparelho de radiografia foi calibrado para $35 \mathrm{kVp}$ e $2 \mathrm{mAs}$, com distância focofilme de $1 \mathrm{~m}$. Como referencial densitométrico nas tomadas radiográficas, utilizou-se um penetrômetro, o qual foi radiografado concomitantemente com os antebraços das cadelas.

Para a realização das leituras densitométricas, foi utilizado um scanner com adaptador para transparências HP Scanjet 4C, para a digitalização das imagens radiográficas e posterior análise por meio do programa computacional analisador de imagens Image ProPlus $^{3}$. A leitura da densidade do osso foi realizada no terço proximal e distal do rádio, na falha óssea produzida.

As inferências estatísticas dos dados de densidade óptica foram avaliadas em esquema de parcelas subdivididas no tempo, considerando como parcelas as cadelas-controles e OSH e os tratamentos hidroxiapatita com alandronato e hidroxiapatita com colágeno e as subparcelas os

\footnotetext{
${ }^{1}$ JHS Laboratório Químico Ltda - Belo Horizonte, Brasil

${ }^{2}$ Shering-Plough Veterinária - São Paulo, Brasil.

${ }^{3}$ Media Cybernetics ${ }^{\circledR}$ - Bethesda, MD, EUA.
}

dias de pós-operatório (0, 30, 60, 90 e 120 dias). Aplicou-se, ainda, o teste de Tukey para comparações múltiplas (Gomes, 1987).

\section{RESULTADOS E DISCUSSÃO}

$\mathrm{Na}$ avaliação clínica, realizada diariamente durante os 20 dias subsequentes à cirurgia, observou-se que todos os animais apresentaram evolução clínica satisfatória, sem alterações sistêmicas que pudessem ser atribuídas aos biomateriais utilizados. Não ocorreram deiscências das suturas ou outra manifestação que demonstrasse reações locais associadas à rejeição do implante, tais como, fístulas ou exsudados purulentos, evidenciando-se, assim, a biocompatibilidade destes produtos, como já descrita por outros pesquisadores (Bell e Beirne, 1988; Pissiotis e Spanberg, 1990; Meraw e Reeve, 1999; Linhares, 2000).

No primeiro dia pós-operatório e durante as primeiras 72 horas, verificou-se em um animal do grupo-controle claudicação moderada do membro torácico esquerdo e em dois animais do grupo OSH claudicação moderada do membro torácico direito. Borges (1998) e Rezende et al. (1998) observaram claudicação em todos os animais nas primeiras 12 horas do período pósoperatório; já Linhares (2000) observou que todos os animais do grupo tratado com COLHAP apresentaram claudicação intensa, durante as primeiras 72 horas do pós-operatório, em decorrência de o limiar de dor desses animais ser mais exacerbado em relação aos demais.

Os resultados dos exames laboratoriais realizados quatro meses pós-OSH revelaram valores de hemograma dentro dos parâmetros normais para ambos os grupos. Muitos pesquisadores afirmaram que, após quatro meses da OSH, existe uma perda significativa de osso e uma notável diminuição do fósforo e do cálcio (Malluche et al., 1986; Malluche et al., 1988; Skolnikov et al., 1992; Chachra et al., 2000). Neste estudo, ao se avaliarem as dosagens de cálcio, fósforo e magnésio séricos, não foram observadas diferenças significativas entre as dosagens do grupo OSH $(10,8 \pm 0,8 \mathrm{mg} / \mathrm{dL}$; $4,6 \pm 1,1 \mathrm{mg} / \mathrm{dL} \quad$ e $\quad 2,2 \pm 0,3 \mathrm{mg} / \mathrm{dL}$, respectivamente), e as do grupo-controle $(10,5 \pm 0,6 \mathrm{mg} / \mathrm{dL} ; 3,7 \pm 0,6 \mathrm{mg} / \mathrm{dL}$ e $2,1 \pm 0,9 \mathrm{mg} / \mathrm{dL}$, respectivamente), indicando que não houve interferência da OSH sobre esses parâmetros. A 
média dos valores da fosfatase alcalina foi significativamente maior no grupo-controle $(130 \pm 38,8 \mathrm{U} / \mathrm{L})$ em relação ao grupo $\mathrm{OSH}$ $(84,3 \pm 23,3 \mathrm{U} / \mathrm{L})$. A fosfatase alcalina, enzima envolvida na formação das células ósseas, é o marcador mais amplamente utilizado na estimativa da taxa de formação óssea, mesmo que essa medida inclua a atividade derivada de outras fontes, tais como fígado e intestino delgado (Stepan et al., 1987), evidenciando provavelmente maior absorção de cálcio pelos animais do grupo-controle.

Todas as cadelas do grupo-controle apresentaram estro durante a realização do experimento.

A Fig. 1A ilustra o exame radiográfico no dia 0 do pós-operátorio, observando-se as falhas ósseas realizadas no terço proximal e distal de ambos os membros. Verificou-se no $30^{\circ}$ dia pósoperatório, em todos os animais do grupocontrole, fechamento total da falha óssea no terço distal do membro direito, onde foi utilizada a HAP-AL. Já no terço proximal, somente quatro animais apresentavam fechamento total e o restante dos animais, fechamento parcial. Comparando com o terço distal do membro esquerdo, onde se utilizou a COL-HAP, quatro cadelas apresentavam fechamento parcial. No terço proximal do mesmo membro, quatro cadelas apresentaram fechamento total e três, fechamento parcial. Analisando estes resultados, pode-se inferir que a HAP-AL é um biomaterial que acelera o processo de cicatrização, por apresentar quimiotactismo pela superfície do osso, diminuindo a reabsorção e aumentado a formação óssea (Pols et al., 1999).

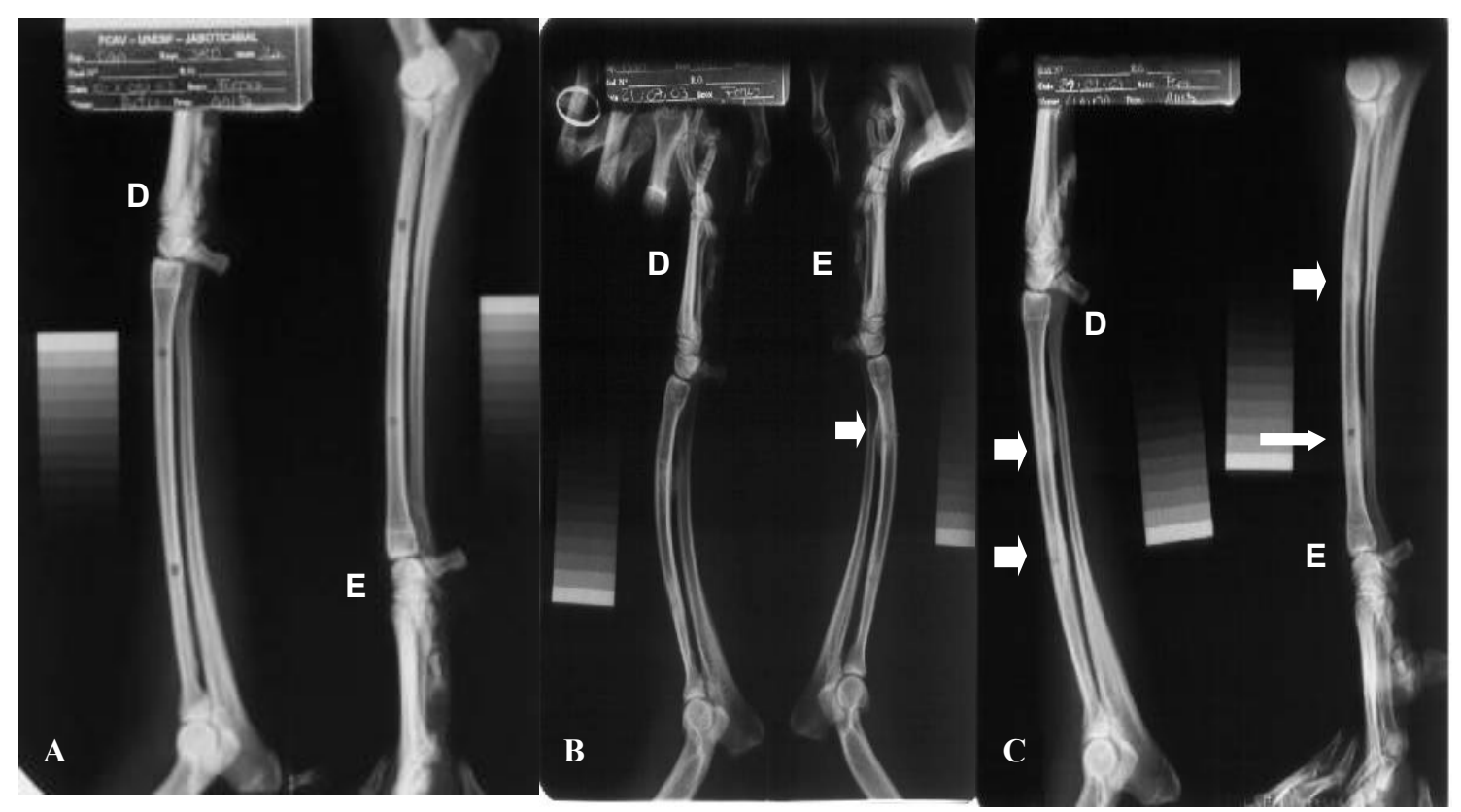

Figura 1. A. Imagem radiográfica em projeção lateromedial do membro torácico direito (D) e esquerdo (E) de uma cadela no dia 0 do pós-operatório. B. Imagem radiográfica em projeção lateromedial no $90^{\circ}$ dia pós-operatório em uma cadela do grupo controle. Observa-se no terço distal do membro torácico esquerdo a remodelação da neoformação do calo ósseo e fechamento parcial da falha óssea (seta). C. Imagem radiográfica em projeção lateromedial no $120^{\circ}$ dia pós-operatório em uma cadela do grupo OSH. Observa-se o fechamento total no terço proximal de ambos membros e no terço distal direito (seta grossa) e fechamento parcial das falhas ósseas do terço distal esquerdo (seta fina).

No grupo OSH, quatro animais apresentaram fechamento total no terço distal do membro direito (HAP-AL), e os demais apresentaram fechamento parcial aos 30 dias do período pósoperatório. Analisando o terço distal do membro esquerdo (COL-HAP), não se observou em nenhum dos pacientes fechamento parcial nem total da falha óssea; já no terço proximal de ambos os membros, verificou-se em cinco cadelas fechamento parcial e em duas 
fechamento total. Notou-se que, nos animais do grupo $\mathrm{OSH}$, o tempo de cicatrização das fraturas foi mais demorado, observações semelhantes foram feitas por Bagi et al. (1992); Skolnikov et al. (1992); Han e Szarzanowicz (1998) ao relatarem que a depleção de estrógenos aumenta a produção de osteoclastos, o que pode interferir na consolidação óssea.

As Tab. 1 e 2 apresentam os achados radiográficos (reação periosteal, remodelação da reação periosteal, neoformação óssea, remodelação da neoformação, fechamento da falha óssea), do terço distal de ambos os membros das cadelas-controle e das OSH (onde houve supressão da atividade ovariana). Os critérios de avaliação utilizados foram, em relação à intensidade, discreta, moderada e severa; e em relação ao fechamento da falha óssea, total ou parcial.

Tabela 1. Avaliação dos parâmetros analisados no rádio de cadelas controle submetidas ao tratamento de hidroxiapatita com alandronato e hidroxiapatita com colágeno, no $30^{\circ}, 60^{\circ}, 90^{\circ}$ e $120^{\circ}$ dias

\begin{tabular}{llcccc}
\hline \multirow{2}{*}{ Resultados radiográficos } & \multicolumn{1}{c}{ Tipo de } & \multicolumn{4}{c}{ Dias } \\
\cline { 2 - 6 } & biomaterial & $30^{\circ}$ & $60^{\circ}$ & $90^{\circ}$ & $120^{\circ}$ \\
\hline Reação periosteal & HAP-Al & +++ & - & - & - \\
\multirow{2}{*}{ Remodelação da reação periosteal } & COL-HAP & ++ & - & - & - \\
\multirow{2}{*}{ Neoformação óssea } & HAP-Al & ++ & ++ & - & - \\
& COL-HAP & ++ & ++ & + & - \\
Remodelação da neoformação & HAP-Al & ++ & +++ & +++ & +++ \\
& COL-HAP & + & + & + & ++ \\
Fechamento da falha óssea & HAP-Al & ++ & +++ & +++ & +++ \\
& COL-HAP & - & + & + & + \\
& HAP-Al & +++ & +++ & +++ & +++ \\
\hline
\end{tabular}

HAP-Al: hidroxiapatita com alandronato; COL-HAP: hidroxiapatita com colágeno.

ausente (-); observado com discreta intensidade (+); observado com moderada intensidade/parcialmente fechada $(++)$; observado com severa intensidade/totalmente fechada $(+++)$.

Tabela 2. Avaliação dos parâmetros analisados no rádio de cadelas submetidas à ovariossalpingohisterectomia e ao tratamento de hidroxiapatita com alandronato e hidroxiapatita com colágeno, no $15^{\circ}$ $20^{\circ}, 30^{\circ}, 60^{\circ}, 90^{\circ}$ e $120^{\circ}$ dias pós-operatórios

\begin{tabular}{llllllc}
\hline \multirow{2}{*}{ Resultados radiográficos } & \multicolumn{1}{c}{ Tipo de } & \multicolumn{5}{c}{ Dias } \\
\cline { 3 - 7 } & biomaterial & $15-20^{\circ}$ & $30^{\circ}$ & $60^{\circ}$ & $90^{\circ}$ & $120^{\circ}$ \\
\hline Reação periosteal & HAP-Al & + & ++ & - & - & - \\
\multirow{2}{*}{ Remodelação da reação periosteal } & COL-HAP & + & + & - & - & - \\
\multirow{3}{*}{ Neoformação óssea } & HAP-Al & - & ++ & ++ & - & - \\
& COL-HAP & - & + & ++ & ++ & + \\
Remodelação da neoformação & HAP-Al & - & ++ & ++ & +++ & +++ \\
& COL-HAP & - & - & + & + & ++ \\
Fechamento da falha óssea & HAP-Al & - & ++ & ++ & +++ & +++ \\
& COL-HAP & - & - & - & + & + \\
& HAP-Al & - & + & ++ & +++ & +++ \\
\hline
\end{tabular}

HAP-Al: hidroxiapatita com alandronato; COL-HAP: hidroxiapatita com colágeno; ausente (-); observado com discreta intensidade $(+)$; observado com moderada intensidade/parcialmente fechada $(++)$; observado com severa intensidade/totalmente fechada $(+++)$.

Avaliando as densidades ósseas (Tab. 3), no $30^{\circ}$ dia do período pós-operatório das cadelas do grupo-controle, verifica-se que a densidade óssea foi maior no terço distal do membro direito onde se implantou a HAP-AL, mas não houve diferença significativa entre os tratamentos ( $\mathrm{P}>0,05)$. Já nos $60^{\circ}, 90^{\circ}$ e $120^{\circ}$ dias do pósoperatório, no terço distal dos membros esquerdos tratados com COL-HAP, a densidade foi significativamente menor, $(\mathrm{P}<0,05)$. 
Tabela 3. Valores densitométricos e desvio-padrão do terço proximal e distal dos membros torácicos das cadelas-controles e submetidas à ovariossalpingo-histerectomia $(\mathrm{OSH})$ tratadas com hidroxiapatita com alandronato e com hidroxiapatita com colágeno no $0,30^{\circ}, 60^{\circ}, 90^{\circ}$ e $120^{\circ}$ dia do período pós-operatório

\begin{tabular}{|c|c|c|c|c|c|c|}
\hline & \multirow{2}{*}{ Tratamento } & \multirow{2}{*}{ Dia zero } & \multicolumn{4}{|c|}{ Dias pós-operatórios } \\
\hline & & & 30 & 60 & 90 & 120 \\
\hline \multirow{4}{*}{$\begin{array}{l}\frac{0}{0} \\
\text { Ü } \\
0 \\
0\end{array}$} & HAP-AL - D D & $3,37 \mathrm{dA} \pm 0,15$ & $11,06 \mathrm{cA} \pm 0,43$ & $13,60 \mathrm{bA} \pm 1,32$ & $15,13 \mathrm{aA} \pm 0,92$ & $16,21 \mathrm{aA} \pm 0,84$ \\
\hline & Controle - D P & $3,03 \mathrm{dA} \pm 0,45$ & $9,78 \mathrm{cAB} \pm 1,14$ & $11,74 \mathrm{bAB} \pm 1,48$ & $13,42 \mathrm{aAB} \pm 1,83$ & $14,56 \mathrm{aA} \pm 1,30$ \\
\hline & Col. HAP - E D & $3,58 \mathrm{cA} \pm 0,18$ & $9,13 \mathrm{bABC} \pm 1,02$ & $9,78 \mathrm{bBC} \pm 1,02$ & $11,17 \mathrm{aCD} \pm 1,68$ & $11,96 \mathrm{aD} \pm 1,90$ \\
\hline & Controle - EP & $3,15 \mathrm{dA} \pm 0,10$ & $10,11 \mathrm{cAB} \pm 1,02$ & $11,69 \mathrm{Bab} \pm 1,85$ & $13,36 \pm 1,80$ & $14,32 \mathrm{aAB} \pm 1,55$ \\
\hline \multirow{4}{*}{$\begin{array}{l}\vec{D} \\
0 \\
0\end{array}$} & HAP-AL - D D & $3,31 \mathrm{eA} \pm 0,25$ & $8,60 \mathrm{dBCD} \pm 0,89$ & $11,19 \mathrm{cB} \pm 1,75$ & $12,71 \mathrm{bBC} \pm 1,90$ & $14,29 \mathrm{aABC} \pm 1,57$ \\
\hline & Controle - D P & $3,10 \mathrm{dA} \pm 0,31$ & $7,50 \mathrm{cCD} \pm 1,34$ & $9,78 \mathrm{bBC} \pm 1,62$ & $10,91 \mathrm{bCD} \pm 1,63$ & $12,27 \mathrm{aBCD} \pm 1,67$ \\
\hline & Col. HAP - E D & $3,49 \mathrm{dA} \pm 0,34$ & $6,92 \mathrm{cD} \pm 1,68$ & $8,31 \mathrm{bc} \pm 1,79$ & $9,49 \mathrm{bC} \pm 0,86$ & $11,27 \mathrm{aD} \pm 1,38$ \\
\hline & Controle - EP & $3,14 \mathrm{dA} \pm 0,20$ & $7,58 \mathrm{cCD} \pm 1,16$ & $9,90 \mathrm{bBC} \pm 1,63$ & $11,01 \mathrm{abCD} \pm 1,72$ & $12,13 \mathrm{aCD} \pm 1,51$ \\
\hline
\end{tabular}

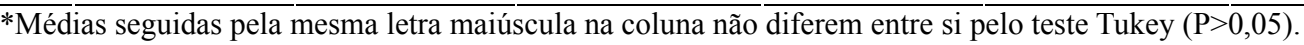

**Médias seguidas pela mesma letra minúscula na linha não diferem entre si pelo teste Tukey $(\mathrm{P}>0,05)$.

HAP-Al: Hidroxiapatita com alandronato; COL-HAP: hidroxiapatita com colágeno; DD: membro direito terço distal; DP: membro direito terço proximal; ED: membro esquerdo terço distal; EP: membro esquerdo terço proximal.

Observa-se, neste experimento, que a densidade óssea foi significativamente maior nas cadelas do grupo-controle $(\mathrm{P}<0,05)$ (Tab. 4). Este resultado confirma o fato de que os estrógenos estimulam a formação do tecido ósseo (Junqueira e Carneiro, 1999), e que a interrupção da função ovariana diminui a massa do osso, incrementando a reabsorção da área endocortical (Bagi et al.,
1992; Souza et al., 2003) e, consequentemente, a diminuição da densidade óssea. Além disso, também se evidenciou em todos os animais de ambos os grupos maior densidade óssea no terço distal onde foi implantada a HAP-AL. Resultado semelhante foi obtido por Rocha (2001) ao observar que o alandronato aumentou a densidade óssea alveolar em cães.

Tabela 4. Valores densitométricos e desvio-padrão dos membros torácicos das cadelas-controles e submetidas à ovariossalpingo-histerectomia (OSH) tratadas com hidroxiapatita com alandronato e com colágeno no $0,30^{\circ}, 60^{\circ}, 90^{\circ}$ e $120^{\circ}$ dia do período pós-operatório

\begin{tabular}{lccccc}
\hline \multirow{2}{*}{ Tratamento } & \multirow{2}{*}{ Dia zero } & \multicolumn{4}{c}{ Dias pós-operatórios } \\
\cline { 3 - 6 } Controle & $3,28 \mathrm{eA} \pm 0,32$ & $10,02 \mathrm{dA} \pm 1,14$ & $11,70 \mathrm{cA} \pm 1,94$ & $13,27 \mathrm{bA} \pm 2,08$ & $14,26 \mathrm{aA} \pm 2,06$ \\
& & & & & \\
OSH & $3,26 \mathrm{eA} \pm 0,31$ & $7,65 \mathrm{~dB} \pm 1,37$ & $9,79 \mathrm{cB} \pm 1,91$ & $11,03 \mathrm{bB} \pm 1,88$ & $12,49 \mathrm{aB} \pm 1,83$
\end{tabular}

*Médias seguidas pela mesma letra maiúscula na coluna não diferem entre si pelo teste Tukey $(\mathrm{P}>0,05)$.

**Médias seguidas pela mesma letra minúscula na linha não diferem entre si pelo teste Tukey $(\mathrm{P}>0,05)$.

No $60^{\circ}$ dia pós-operatório, nas cadelas do grupocontrole, observou-se o remodelamento da reação periosteal, e constatou-se espessamento da cortical com maior intensidade no membro direito, onde foi colocada a HAP-AL. Obteve-se, portanto, resultados similares aos de Meraw e Reeve (1999) que utilizaram implantes dentários em cães com uma camada de alandronato e Yaffe (1997; 1999) que utilizou este mesmo biomaterial na mandíbula de ratos, estes autores concluíram que o alandronato aumentou a neoformação óssea.
Aos 90 dias pós-operatórios, o calo ósseo neoformado e remodelado no terço distal do membro direito apresentou-se com aumento da radiopacidade. Observou-se o fechamento parcial da falha óssea no terço distal do membro esquerdo, em duas cadelas do grupo OSH e uma cadela do grupo-controle (Fig. 1B).

Evidenciou-se no $120^{\circ}$ dia pós-operatório remodelação da neoformação óssea e fechamento total da falha óssea do terço proximal de ambos os membros e no terço distal do membro direito dos dois grupos. No terço distal esquerdo, uma cadela do grupo-controle e duas do grupo $\mathrm{OSH}$ 
apresentavam fechamento parcial (Fig. 1C). Estes achados não diferem das observações feitas por Linhares (2000), pois no $120^{\circ}$ dia do pósoperatório, os animais do grupo tratado com COL-HAP mostraram radiopacidade igual à do osso somente próxima às bordas do defeito, com áreas radiotransparentes no centro da falha, não apresentando preenchimento total do defeito ósseo. Ao contrário dessa observação (Bell e Beirne, 1988), não encontraram alterações no padrão de reparação óssea, quando compararam a hidroxiapatita pura e a hidroxiapatita associada ao colágeno. Contudo Pissiotis e Spangberg (1990) constataram que o tecido ósseo de reparação mostrava-se mais imaturo quando o implante se constituía de colágeno associado à hidroxiapatita. Baseado nestas observações, pode-se inferir que a associação do colágeno com a hidroxiapatita parece diminuir a capacidade osteocondutora do biomaterial na reparação óssea, o que também foi observado por Linhares (2000).

No grupo OSH, verificou-se que aos 30, 60, 90 e 120 dias do pós-operatório, a densidade óssea foi maior $(\mathrm{P}<0,05)$ no terço distal do membro direito onde foi implantada a HAP-AL (Tab. 3). Evidenciou-se nesta pesquisa que a densitometria óptica é uma técnica que elimina possíveis interpretações subjetivas obtidas durante a avaliação radiográfica como foi descrito por Glüer e Genant (2002).

\section{CONCLUSÕES}

A hidroxiapatita associada ao alandronato acelera o processo de reparação óssea, ao contrário da hidroxiapatita associada ao colágeno; a interrupção da função ovariana produz um retardo na cicatrização do tecido ósseo; e a densitometria óptica é uma técnica não invasiva adequada para a avaliação pós-cirúrgica da consolidação óssea, que permite eliminar interpretações subjetivas obtidas durante a avaliação radiográfica, além de permitir analisar as diferenças qualitativas e quantitativas na reação periosteal.

\section{REFERÊNCIAS BIBLIOGRÁFICAS}

BAGI, C.M.; MILLER, S.C.; BOWMAN, B.M. et al. Differences in cortical bone in overloaded and underloaded femurs from ovariectomized rats: comparison of bone morphometry with torsional testing. Bone, v.13, p.35-40, 1992.

BAKOS, D.; SOLDAN, M.; HERNANDEZFLUENTES, I. et al. Hydroxyapatite-collagenhyaluronic-acid composite. Biomaterials, v.20, p.191-195, 1999.

BELL, R.; BEIRNE, O.R. Effect of hydroxyapatite, triclacium phosphate, and collagen on the healing of defects in the rat mandibule. J. Oral Maxillofac. Surg., v.46, p.589-894, 1988.

BORGES, A.P.B. O efeito da hidroxiapatita sintética na regeneração óssea de defeito provocado experimentalmente no terço proximal da tíbia de cães (canis familiares). Estudo clínico-cirúrgico, radiológico e histológico por microscopia de luz e microscopia eletrônica de retrodispersão. 1998. 111f. Tese (Doutorado) Escola de Veterinária, Universidade Federal de Minas Gerais, Belo Horizonte, 1998.

CHACHRA, D.; LEE, J.M.; KASRA, M. et al. Differential effects of ovariectomy on the mechanical properties of cortical and cancellous bone in rat femora and vertebrae. Biomed. Sci. Instrum., v.36, p.123-128, 2000.

DU, C.; CUI, F.Z.; FENG, Q.L. et al. Therapeutic applications of implantable drug delivery systems. J. Pharmacol. Toxicol. Met., v.40, p.1-12, 1998.

GLÜER, C.; GENANT, H. Métodos atuais de detecção da densidade mineral óssea, [200-]. Disponível em: http://www.institutoradiologia.com.br/artigos/artigo2.htm. Acessado em: 05 out. 2002

GOMES, F.P. Curso de estatística experimental. 12.ed. Piracicaba: Nobel, 1987.

HAN, S.M.; SZARZANOWICZ, T.E. Effect of ovariectomy and calcium deficiency on the ultrasound velocity, mineral density and strength in the rat femur. Clin. Biomech., v.13, p.480-484, 1998.

JUNQUEIRA, L.C.; CARNEIRO, J. Histologia básica. 9.ed. Rio de Janeiro: Guanabara Koogan, 1999.

LINHARES, F.K. Avaliação clínico-cirúrgica, radiológica e histológica da reparação óssea após utilização de HAP-91, COL.HAP-91 e INT.HAP-91 em defeito provocado experimentalmente na tíbia de cães. 2000. 37f. 
Monografia (Especialização) - Universidade Federal de Viçosa, Viçosa, MG.

MALLUCHE, H.H.; FAUGERE, M.C.; RUSH, $M$. et al. Osteoblastic insufficiency is responsible for maintenance of osteopenia after loss of ovarian function in experimental Beagle dogs. Endocrinology, v.119, p.2649-2654, 1986.

MALLUCHE, H.H.; FAUGERE, M.C.; RUSH, M. et al. 1,25-Dihidroxyvitamin D3 corrects bone loss but suppresses bone remodeling in ovariohysterectomized Beagle dogs. Endocrinology, v.122, p.1998-2006, 1988.

MANN, S.; WEBB, J.; WILLIAMS, R.J.P. Biomineralization: chemical and biochemical perspectives. New York: VCH Publishers, 1989.

MASARACHIA, P. Comparison of the distribution of $3 \mathrm{H}$-alendronate and $3 \mathrm{H}$-etidronato in rat and mouse bones. Bone, v.19, p.281-290, 1996.

MERAW, S.J.; REEVE, C.M. Qualitative analysis of peripheral peri-implant bone and influence of alendronate sodium on early bone regeneration. J. Periodontol., v.70, p.1228-1233, 1999.

PISSIOTIS, E.; SPANBERG, L.S.W. Biological evaluation of collagen gels containing calcium hydroxide and hydroxyapatite. J. Endodontol., v.16, p.486-473, 1990.

POLS, H.P.; FELSENBERG, D.; HANLEY, D.Z. Multinational, placebo-controlled, randomized trial of the effects of alendronate on bone density the FOSIT study. Osteoporose, v.23, p.461-468, 1999.

REZENDE, C.M.F.; BORGES, A.B.P.; BERNIS, W.O. Aspectos clínico-cirúrgicos e radiográficos da hidroxiapatita sintética na diáfise proximal da tíbia de cães. Arq. Bras. Med. Vet. Zootec., v.50, p.537-545, 1998.
ROCHA, M. Clinical and radiological improvement of periodontal disease in patients with type 2 diabetes mellitus treated with alendronate: a randomized, placebo-controlled trial. J. Periodontol., v.72, p.204-209, 2001.

SKOLNIKOV, A.A.; KODENTSOVA, V.M.; SPIRICHEV, V.B. The calcium and phosphorus content of the bone tissue in ovariectomized rats with a fracture of the tibia: the effect of vitamin $\mathrm{K}$ and vitamin D metabolites. Fisiology, v.38, p.29-36, 1992.

SOUZA, A.; BORGES, J.; RAGI, S. et al. Ultrassonometria óssea, [200-]. Disponível em: $<$ http://www.fertile.com.br/pages/folder11/arti04 -040700. Acessado em: 05 set. 2003

STEPAN J.; POSPICHAL, J.; PRESL, J. et al. Bone loss and biochemical indices of bone remodeling in surgically induced postmenopausal women. Bone, v.8, p.279-284, 1987.

WHEELER, D.L.; ESCHBACH, E.J.; MONTFORD, M.J. et al. Mechanical strength of fracture callus in osteopenic bone at different phases of healing. J. Orthop. Trauma, v.14, p.8692, 2000 .

YAFFE, A. Disposition of alendronate following local delivery in rat jaw. J. Periodontol., v.71, p.893-895, 1999.

YAFFE, A. Local delivery of na amino bisphosphonato prevents the resorptive phase of alveolar bone following mucoperiosteal flap surgery in rats. J. Periodontol., v.68, p.884-889, 1997. 